\title{
Chaotic Analysis and Machine Learning Diagnosis of Herringbone-grooved Journal Gas Bearing System
}

\author{
Cheng-Chi Wang, ${ }^{1 *}$ Cai-Wan Chang-Jian, ${ }^{2}$ and Ping-Hung Wang ${ }^{3}$ \\ ${ }^{1}$ Graduate Institute of Precision Manufacturing, National Chin-Yi University of Technology, \\ No. 57, Sec. 2, Zhongshan Rd., Taiping Dist., Taichung 411030, Taiwan \\ ${ }^{2}$ Department of Mechanical and Automation Engineering, I-Shou University, \\ No. 1, Sec. 1, Syuecheng Rd., Dashu District, Kaohsiung City 84001, Taiwan \\ ${ }^{3}$ Department of Mechanical Engineering, National Chin-Yi University of Technology, \\ No. 57, Sec. 2, Zhongshan Rd., Taiping Dist., Taichung 411030, Taiwan
}

(Received June 12, 2021; accepted November 8, 2021)

Keywords: herringbone-grooved journal gas bearing, chaos, maximum Lyapunov exponent, GoogLeNet, ResNet-50

In the modern precision machinery industry, although gas bearings (GBs) are commonly used in ultraprecision machining, precision measurement, and high-speed cutting, the irregular motion of a GB, i.e., nonlinear behavior, is often found under high-speed rotation owing to rotor eccentricity and gas characteristics. Herringbone-grooved journal gas bearings (HJGBs) have been increasingly used in mechanisms requiring precision rotation owing to their high stability and the better support force applied to the rotor than traditional GBs. However, the strong nonlinearity of the function describing the air film pressure and the dynamic problems of actual bearing systems, including inappropriate rotational speed, unbalanced gas supply, and improper bearing design, will cause the nonperiodic or chaotic motion and instability of the rotor-bearing system under some conditions. Such irregular motion will damage machines in some situations. To determine the conditions that cause the rotor of HJGBs to undergo nonperiodic motion, and thereby prevent irregular vibration, we apply embedded piezo vibration sensors in an HJGB system to investigate in detail the relevant bearing characteristics and verify the results. To examine the nonlinear behavior of rotors, Poincaré maps and the maximum Lyapunov exponent are used to detect the vibration signal from piezo sensors, and GoogLeNet and ResNet-50 are used to identify the type of vibration behavior. The results of rotor behavior diagnosis show that the accuracies of GoogLeNet and ResNet-50 were 100 and 99.7\%, respectively, i.e., GoogLeNet outperformed ResNet-50. This paper provides a diagnosis of the occurrence of chaos in an HJGB and a means of inhibiting nonperiodic motion to reduce the system loss caused by irregular vibration. 


\section{Introduction}

In 1994, Bonneau and Absi ${ }^{(1)}$ studied the characteristics of a herringbone-grooved journal gas bearing (HJGB) using the finite element method. These characteristics included the stability, the pressure distribution, and the effects of some important parameters on the system. Several researchers have analyzed the characteristics of an HGJB in the spindle motor of a computer hard disk drive. Jang and $\mathrm{Kim}^{(2)}$ calculated dynamic coefficients in an HGJB and a thrust bearing, considering five degrees of freedom for a general rotor-bearing system. Wang and coworkers $^{(3,4)}$ analyzed the motion of a rotor and journal center in the horizontal and vertical directions on the basis of the performance of a flexible rotor supported with an HJGB system using the finite difference method (FDM) and successive over-relaxation (SOR). They observed the dynamic behavior of the rotor and journal center in the HJGB system under different conditions by root locus analysis and using the Poincaré map, spectral response, and bifurcation diagram. They found that periodic, quasi-periodic, or subharmonic motion, and even chaotic motion, arose in the rotor and journal center under different operating conditions. ${ }^{(5)}$ The effect of chaotic motion on an air film bearing system is unpredictable and it must be avoided because it is especially dangerous for precision bearing systems. Therefore, the main objective of this study is to analyze the dynamic behavior of HJGB systems under different operating conditions and use machine learning (ML) methods to identify rotor behavior and predict whether the rotor has aperiodic behavior. There are many different ML models that can be used to identify rotor behaviors: multilayer perceptron (MLP) ${ }^{(6)}$ support vector machine $(\mathrm{SVM}),{ }^{(7)}$ decision tree $(\mathrm{DT}){ }^{(8)}$ and random forest (RF). ${ }^{(9)}$ However, they have rarely been used to identify Poincaré maps of rotor behavior.

\section{System Modeling of HJGB}

Figure 1 shows the model of an HJGB, whose major groove sits on the rotor. We make the following assumptions about the HJGB:

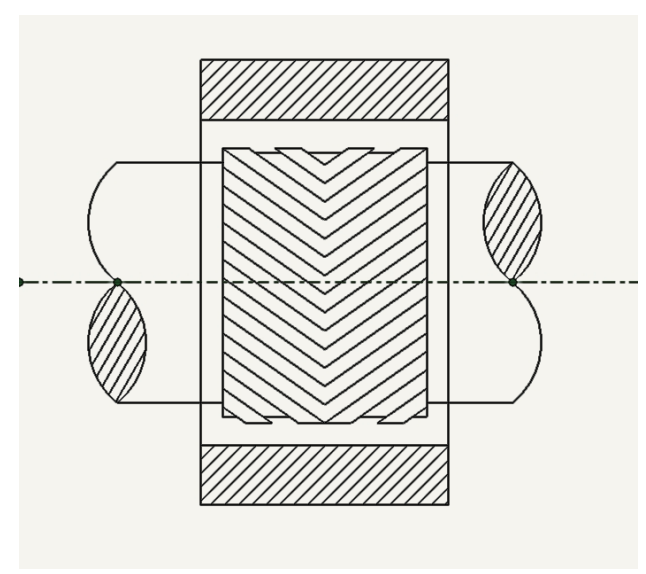

Fig. 1. Model of HJGB. 
- The air film flows at a constant temperature.

- The gas viscosity is constant and remains unchanged irrespective of pressure and temperature changes.

- The gas inflow and outflow at both ends of the bearing are disregarded.

The fluid pressure and air film distribution inside the HJGB must satisfy the following Reynolds equations:

$$
\begin{gathered}
\frac{\partial}{\partial \bar{x}}\left(p h^{3} \frac{\partial p}{\partial \bar{x}}\right)+\frac{\partial}{\partial \bar{z}}\left(p h^{3} \frac{\partial p}{\partial \bar{z}}\right)=12 \mu \frac{\partial}{\partial t}(p h)+6 R \dot{\theta} \mu \frac{\partial}{\partial \bar{x}}(p h), \\
h_{g r}=C_{g}+C_{r}+e_{x} \cos \bar{\theta}+e_{y} \sin \bar{\theta}, \\
h_{r i}=C_{r}+e_{x} \cos \bar{\theta}+e_{y} \sin \bar{\theta}, \\
\frac{\partial h}{\partial t}=\dot{e}_{x} \cos \bar{\theta}+\dot{e}_{y} \sin \bar{\theta} .
\end{gathered}
$$

Equations (2) and (3) represent the air film thicknesses of the groove area $\left(h_{g r}\right)$ and nongroove area $\left(h_{r i}\right)$, respectively.

\section{ML Diagnosis}

In the diagnosis of rotor dynamic behaviors, an accelerator is applied to the rotor to sense its vibration and Poincaré maps are used with ML for the image-based recognition of four behaviors, i.e., types of motion: T-period, 4T-cycle, quasi-periodic, and chaotic behaviors. The raw Poincaré maps have $1476 \times 1181$ pixels. To reduce the time required for the diagnosis, the initial pixel values were input to GoogLeNet and ResNet-50 to reduce the number of Poincaré map pixels to $224 \times 224$. In addition, maps were categorized according to the above four behaviors. To strengthen image training models, images were treated by rotation, enlargement, miniaturization, grid addition, reflection, and other transformations, and 90 images from each treatment were selected as training models.

In ML classification, elements in the confusion matrix were used to calculate the accuracy, precision, recall, and F1 score. ${ }^{(10)}$ True positive (TP), true negative (TN), false positive (FP), and false negative (FN) were used to determine the performance of the model as shown in Fig. 2. FP and FN indicate type I and type II errors, respectively, and are emphasized in ML because these errors can help the adjustment of the model to improve its performance. 


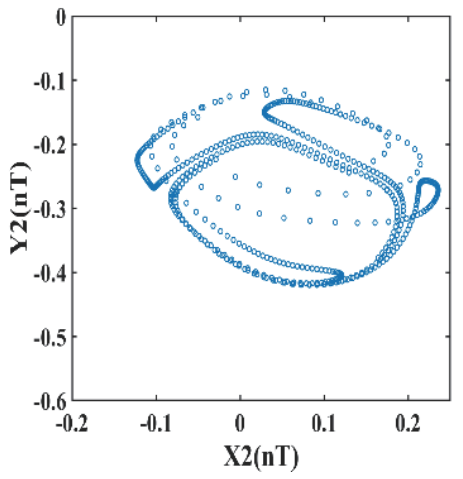

(a)

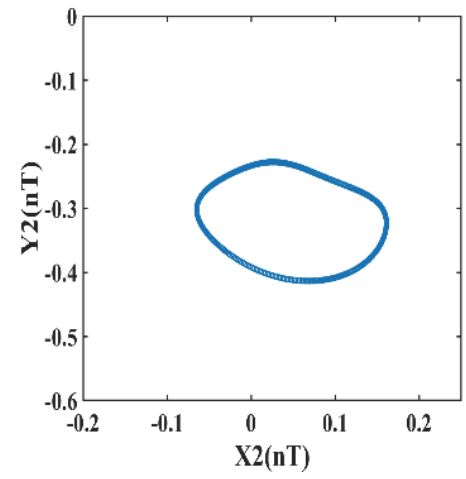

(b)

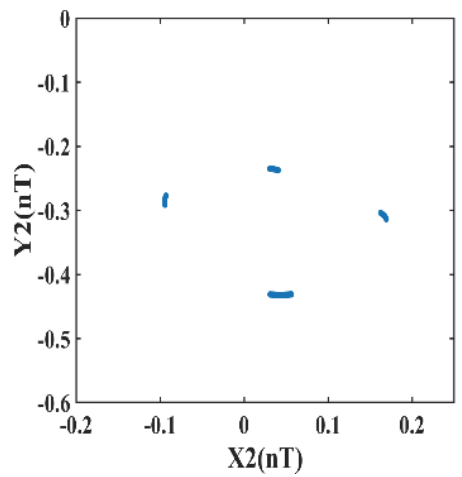

(c)

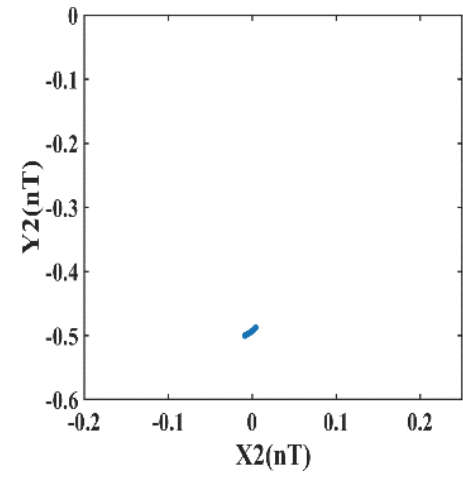

(d)

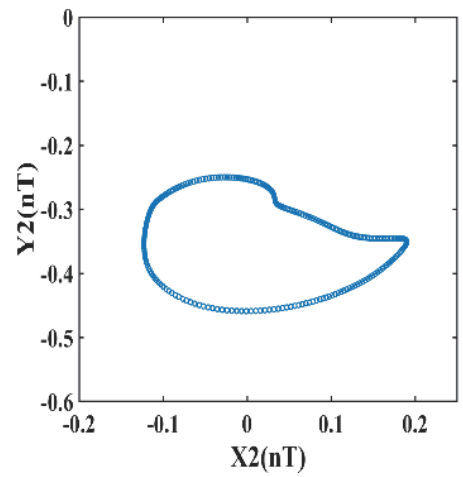

(e)

Fig. 2. (Color online) Dynamic behavior for rotor masses of (a) 7.19, (b) 7.20, (c) 7.63, (d) 9.07, and (e) $9.09 \mathrm{~kg}$.

\section{Results and Discussion}

\subsection{Analysis of rotor dynamic behaviors}

The effects of changes in the rotor mass on the dynamic behaviors of the bearing were observed for a fixed rotational speed (rpm). In this paper, a nondimensional time interval was used. Poincaré maps were calculated and the behaviors, including periodic, quasi-periodic, and chaotic motion, were determined with the acquired rotor motion using sensors. An accelerator was mounted on the housing of the spindle to detect the vibration of the rotor under different operating parameters. Calculations were performed on the signals obtained from the sensors to form the Poincaré maps.

As shown by the Poincaré maps in Fig. 2, when the rotor mass was $7.19 \mathrm{~kg}$, the system was chaotic. When the rotor mass was increased to $7.20 \mathrm{~kg}$, a closed loop was formed by many discrete points, as shown by the Poincaré map, revealing that the system was in a quasi-periodic motion state. When the rotor mass was increased to 7.63 and $9.07 \mathrm{~kg}$, the Poincaré maps contained four discrete points and one discrete point, indicating 4T-subharmonic and T-periodic motion, respectively. When the rotor mass was further increased to $9.09 \mathrm{~kg}$, the dynamic orbit of the rotor became unstable. The Poincaré map also showed a closed loop, indicating quasiperiodic motion. 


\subsection{Diagnosis of rotor dynamic behaviors}

Although the above results show that the Poincaré map can clearly reveal the type of rotor dynamic behavior, the Poincaré map is also used in the image-based diagnosis of motion. We diagnosed images and compared the results with those obtained by GoogLeNet and ResNet-50. The same data set was used for the same bearing system parameters to compare their functions using the confusion matrix. The results show that the accuracy of ResNet-50 in diagnosing quasi-periodic motion is $99.9 \%$, higher than the value of $95.6 \%$ for GoogLeNet, as shown in Figs. 3 and 4. However, GoogLeNet has a higher accuracy than ResNet-50 in diagnosing other types of motion.

The confusion matrix shows that GoogLeNet successfully diagnosed all 360 samples in the data set with an overall accuracy of $100 \%$, and ResNet-50 made only one error (T-periodic) in the $4 \mathrm{~T}$ diagnosis and had an overall accuracy rate of $99.7 \%$, as shown in Fig. 5. Table 1 shows a comparison of their performance characteristics in image diagnosis, with precision, sensitivity, and F1 score as indicators. The results show that GoogLeNet outperforms ResNet-50 in all indicators.

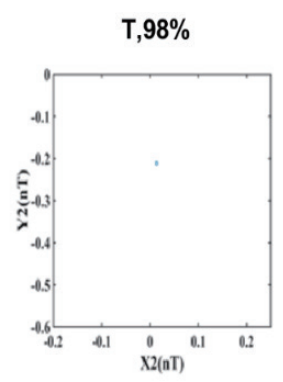

(a)

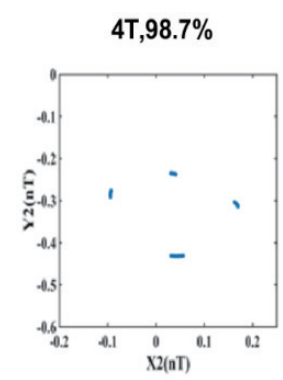

(b)

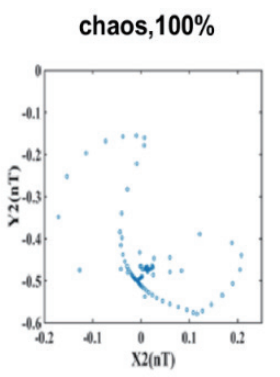

(c)

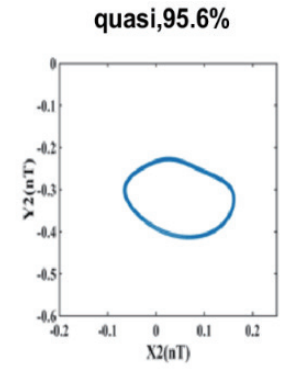

(d)

Fig. 3. (Color online) Recognition of the Poincaré map using GoogLeNet: (a) 1T, (b) 4T, (c) chaotic, and (d) quasiperiodic.

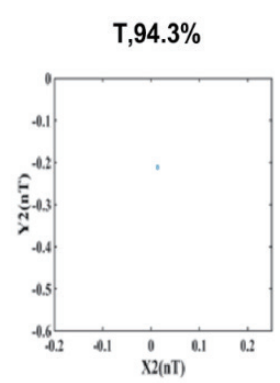

(a)

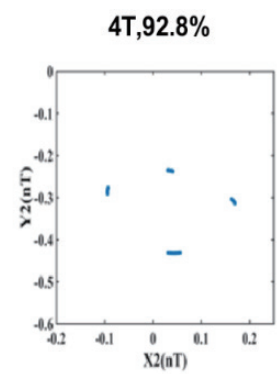

(b)

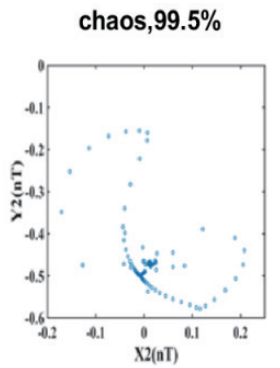

(c)

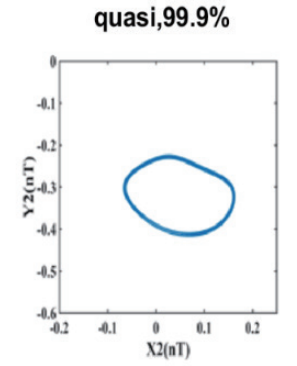

(d)

Fig. 4. (Color online) Recognition of the Poincaré map using ResNet-50: (a) 1T, (b) 4T, (c) chaotic, and (d) quasiperiodic. 


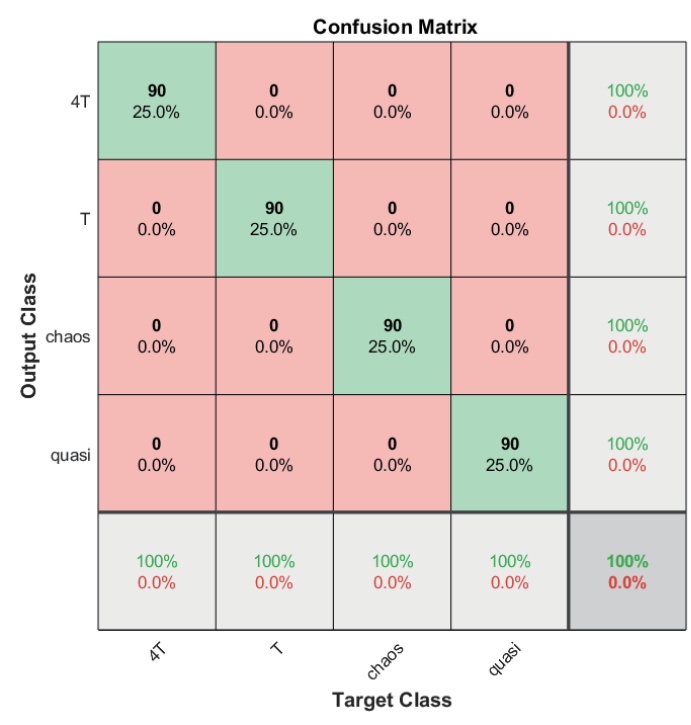

(a)

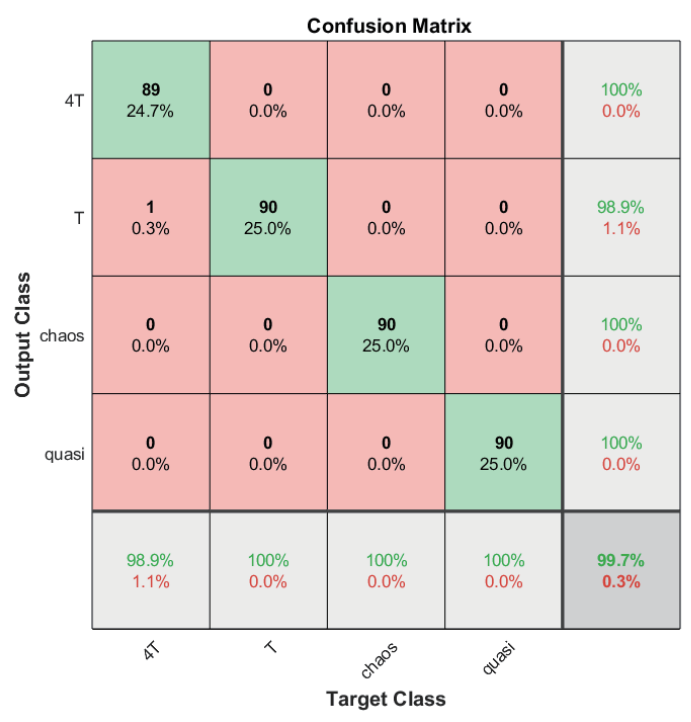

(b)

Fig. 5. (Color online) Confusion matrix for Poincaré map identification using (a) GoogLeNet and (b) ResNet-50.

Table 1

Comparison of various performance indicators of Poincaré map identification with GoogLeNet and ResNet-50.

\begin{tabular}{lccc}
\hline Type & Indicator & GoogLeNet & ResNet-50 \\
\hline \multirow{4}{*}{$1 \mathrm{~T}$} & Precision & 1 & 1 \\
& Sensitivity & 1 & 1 \\
\hline \multirow{3}{*}{$4 \mathrm{~T}$} & F1 score & 1 & 1 \\
& Precision & 1 & 0.989 \\
& Sensitivity & 1 & 0.989 \\
Quasi-periodic & F1 score & 1 & 0.989 \\
& Precision & 1 & 1 \\
& Sensitivity & 1 & 1 \\
Chaotic & F1 score & 1 & 1 \\
& Precision & 1 & 1 \\
& Sensitivity & 1 & 1 \\
\hline
\end{tabular}

\section{Conclusions}

In addition to analyzing the nonlinear dynamic behaviors of an HJGB, we diagnosed rotor behaviors with a $\mathrm{CNN}$ and made dynamic predictions of rotor behaviors with regression analysis to effectively determine and predict chaotic and nonperiodic behaviors. The analysis of the change in rotor mass shows that the behaviors of the system are diverse and include single periodic, 4-periodic, quasi-periodic, and chaotic behaviors. The confusion matrix and various performance indicators, including precision, sensitivity, and F1 score, reveal that the effectiveness of diagnosis with GoogLeNet outperforms that of ResNet-50. Predictions of rotor 
behavior are made to establish the mass range of the motor in which chaotic motion will occur. This study can be used as an important reference for the real-time monitoring of mechanisms with a high rotational speed in the future.

\section{Acknowledgments}

This research was funded by the Ministry of Science and Technology, Taiwan (grant numbers MOST 107-2221-E-167-006, 110-2221-E-167-019, and 110-2221-E-167-019).

\section{References}

1 D. Bonneau and J. Absi: ASME J. Tribol. 116 (1994) 698. https://doi.org/10.1115/1.2927320

2 G. H. Jang and Y. J. Kim: ASME J. Tribol. 121 (1999) 499. https://doi.org/10.1115/1.2834095

3 C. C. Wang: Appl. Math. Modell. 47 (2017) 859. https://doi.org/10.1016/j.apm.2016.08.014

4 C. C. Wang, R. M. Lee, H. T. Yau, and T. E. Lee: J. Low Freq. Noise Vibr. Act. Control. 38 (2019) 1404. https:// doi.org/10.1177/1461348418792737

5 C. C. Wang and C. J. Lin: Symmetry 12 (2020) 1521. https://doi.org/10.3390/sym12091521

6 F. M. M. Mokbal, W. Dan, A. Imran, L. Jiuchuan, F. Akhtar, and W. Xiaoxi: IEEE Access 7 (2019) 100567. https://doi.org/10.1109/ACCESS.2019.2927417

7 F. Alamdar, F. S. Mohammadi, and A. Amiri: IEEE Access 7 (2019) 22260. https://doi.org/10.1109/ ACCESS.2019.2897891

8 X. Wang and F. Liu: IEEE Access 8 (2020) 12105. https://doi.org/10.1109/ACCESS.2020.2965963

9 A. Javeed, S. Zhou, L. Yongjian, I. Qasim, A. Noor, and R. Nour: IEEE Access 7 (2019) 180235. https://doi. org/10.1109/ACCESS.2019.2952107

10 C. C. Wang and Y. Q. Zhu: Symmetry 13 (2021) 1445. https://doi.org/10.3390/sym13081445 